\title{
Adrenocortical adenoma with myelolipomatous metaplasia: a potential diagnostic pitfall: a case report and review of the literature
}

\author{
Mohammad Hossein Anbardar ${ }^{1,2}$, Neda Soleimani ${ }^{1,2^{*}}$, Saman Nikeghbalian ${ }^{3,4}$ and Maryam Mohebbi ${ }^{1}$
}

\begin{abstract}
Background: Adrenal incidentalomas are often found during investigation for another tumor or unrelated problems. Except for adrenal myelolipoma (second most common primary adrenal incidentaloma following adrenocortical adenomas), adrenal lipomatous tumors are uncommon generally and are often described as case reports in the literature. Since the amount of fat is variable, without the help of advanced imaging techniques, some adrenal lipomatous tumors may be misdiagnosed before pathologic examination. Herein, we report a case of adrenal adenoma with myelolipomatous metaplasia that was excised as a periceliac mass in the setting of recurrent pancreatic cyst.
\end{abstract}

Case report: A 45-year-old Iranian woman with hypertension and end-stage renal disease presented with recurrence of a pancreatic cyst (previous pathologic report was mucinous cyst adenoma). During exploratory laparotomy, the mentioned pancreatic cyst was tightly attached to the stomach and jejunum. There was also a periceliac round rubbery lesion (firstly diagnosed by endoscopic ultrasound) that was excised for ruling out malignancy. Histologic examination of the periceliac mass was found to be adrenocortical adenoma with foci of myelolipomatous metaplasia. The pancreatic cyst histology was just a pseudocyst.

Conclusion: Our case highlights the significance of complete evaluation of incidental findings before surgical intervention, even in the setting of another primary tumor. Myelolipoma and myelolipomatous change (metaplasia) are two different entities. Although very similar as to pathogenesis, there are still some differences.

Keywords: Adrenal adenoma, Adrenal myelolipoma, Myelolipomatous metaplasia

\section{Introduction}

Adrenal incidentalomas are often found during investigation for another tumor or unrelated problems. In general, most adrenal incidentalomas (70-80\%) are benign adenomas that cause no symptoms. Differential diagnosis consists of pheochromocytoma, adrenocortical carcinoma, lymphoma, and metastases from various malignancies.

*Correspondence: neda_soleimani61@yahoo.com; soleimani_n@sums.ac.ir

${ }^{1}$ Department of Pathology, Shiraz Medical School, Shiraz University of Medical Sciences, Shiraz, Iran

Full list of author information is available at the end of the article
Adrenal lipomatous tumors (myelolipoma, lipoma, teratoma, angiomyolipoma, and adrenocortical tumors with lipomatous and myelolipomatous metaplasia) should also be included [1-5]. Since the amount of fat is variable in lipomatous tumors, in the absence of advanced imaging techniques, they may be misdiagnosed as malignant lesions before pathologic evaluation [6]. Herein, we report a case of adrenal adenoma with myelolipomatous metaplasia that was excised in the setting of recurrent pancreatic neoplasm. original author(s) and the source, provide a link to the Creative Commons licence, and indicate if changes were made. The images or other third party material in this article are included in the article's Creative Commons licence, unless indicated otherwise in a credit line to the material. If material is not included in the article's Creative Commons licence and your intended use is not permitted by statutory regulation or exceeds the permitted use, you will need to obtain permission directly from the copyright holder. To view a copy of this licence, visit http://creativecommons.org/licenses/by/4.0/. The Creative Commons Public Domain Dedication waiver (http://creativeco mmons.org/publicdomain/zero/1.0/) applies to the data made available in this article, unless otherwise stated in a credit line to the data. 


\section{Case report}

A 45-year-old Iranian woman was transferred to our center to be evaluated for abdominal pain and recurrence of a pancreatic cyst. One year ago, she underwent central pancreatectomy and cholecystectomy at another center because of incidental finding of a large pancreatic cyst, and the pathologic report was benign mucinous cyst adenoma. Then, she presented with upper abdominal pain, mildly elevated carcinoembryonic antigen (CEA) level, and recurrence of pancreatic cyst, which were found on follow-up. She was a case of hypertension (HTN) and end-stage renal disease (ESRD) for 5 years due to idiopathic nephrotic syndrome, and she was taking prednisolone, metoral, and amlodipine. She was dialyzed twice a week. She was a married housekeeper lady, G2P2A0L2, without any history of smoking or alcohol consumption. She had normal menstruation, and there was not any history of abdominal cramp, polyuria, polydipsia, polyphagia, and recent weight gain. The patient's psychologic and family history was not significant. On physical examination, blood pressure was $130 / 80 \mathrm{mmHg}$ with normal pulse rate and temperature, 76 beats per minute and $37.1{ }^{\circ} \mathrm{C}$, respectively. Her body mass index (BMI) was 24.5. Chest examination was unremarkable, and the abdomen was soft with no organomegaly. She had normal face and body fat distribution without any hirsutism or pink stretch mark on the abdomen and trunk. There was no significant problem on neurologic examination.

The patient had high blood urea nitrogen (BUN) and creatinine, $27(6-20) \mathrm{mg} / \mathrm{dl}$ and $4.2(0.6-1.3) \mathrm{mg} / \mathrm{dl}$, respectively. Serum fasting blood sugar $(96 \mathrm{mg} / \mathrm{dl})$ and complete blood count $(\mathrm{CBC})$ [white blood cell (WBC):
$10.8 \times 10^{3} / \mu \mathrm{l}$, hemoglobin: $13.2 \mathrm{~g} / \mathrm{dl}$, and platelet count: $\left.224 \times 10^{3} / \mu \mathrm{l}\right]$ were within normal ranges. Liver enzymes including aspartate aminotransferase (AST), aspartate aminotransferase (ALT), and alkaline phosphatase (ALP) were 23(3-40), 21(3-40), and 146(80-306) IU/L respectively. Abdominal sonography showed a large cystic lesion in the body and tail of the pancreas. In addition to pancreatic cyst (measuring $10 \times 8 \times 4 \mathrm{~cm}$ ), endoscopic ultrasound discovered a $2 \times 2 \times 2 \mathrm{~cm}$ solid mass at the left periceliac area. Exploratory laparotomy revealed the mentioned pancreatic cyst with tight attachment to the posterior wall of the stomach and jejunum and the periceliac lesion with suspicious attachment to the left kidney. For ruling out malignancy, the specimens of distal pancreatectomy, splenectomy, and periceliac lesion excision were sent for pathologic evaluation. Gross sectioning of the pancreas showed a hemorrhagic cyst with $1 \mathrm{~cm}$ maximum wall thickness. The periceliac lesion was a round creamy yellow solid firm mass with heterogeneous cut surface.

On microscopy, sections of the periceliac mass showed an adrenocortical adenoma (ACA) with multifocal areas of adipose tissue admixed with bone marrow elements (including megakaryocytes), features in keeping with ACA (approved by immunohistochemistry) with myelolipomatous change, instead of malignancy (Figures 1,2). The pancreatic cyst histology was just a pseudocyst (Figure 3). During hospital admission, the patient received the previously prescribed medication with the same dosage in addition to a short-acting benzodiazepine (oxazepam $10 \mathrm{mg}$ per night)as a sedative. In a follow-up visit 2 weeks later, the patient showed improvement in her

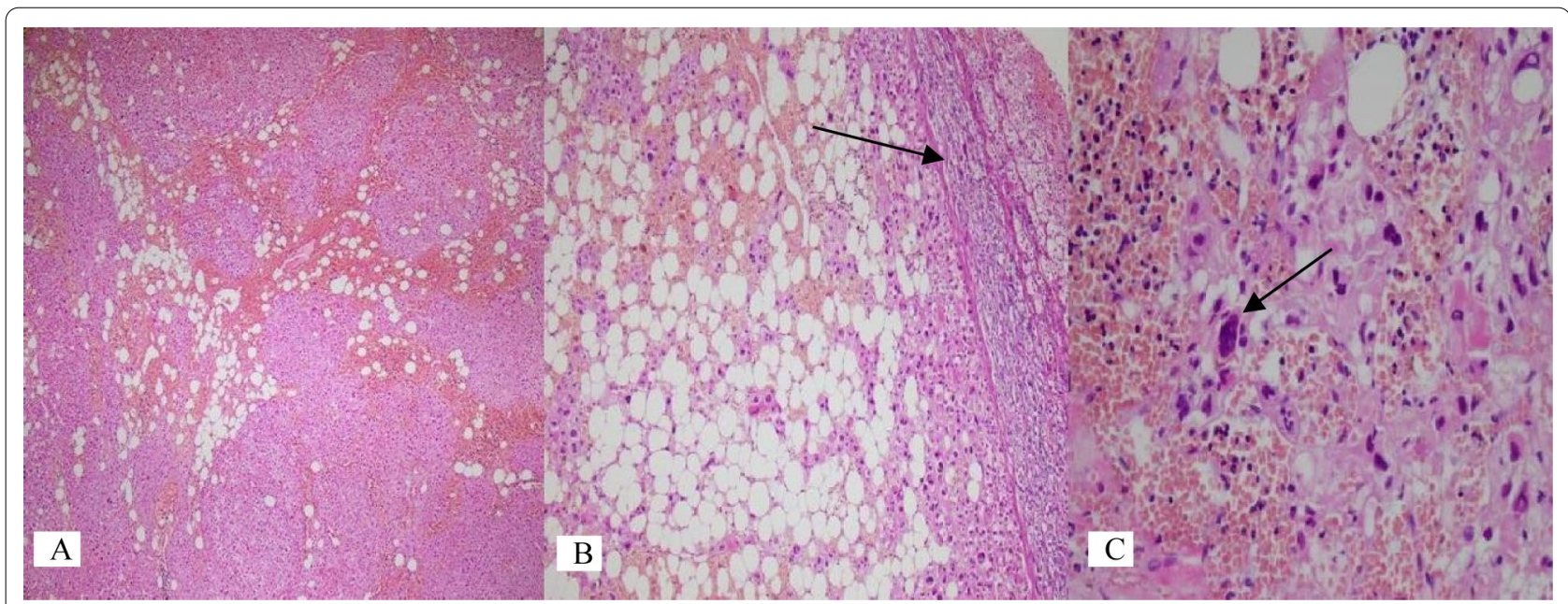

Fig. 1 Histological examination. A Adrenocortical neoplasm intermixed with adipose tissue (hematoxylin and eosin $\times 40$ ). B ACA with myelolipomatous metaplasia and a thin rim of normal adrenal gland (hematoxylin and eosin $\times 100$ ). C Myelolipomatous metaplasia with bone marrow elements including megakaryocyte (arrow) (hematoxylin and eosin $\times 400$ ) 


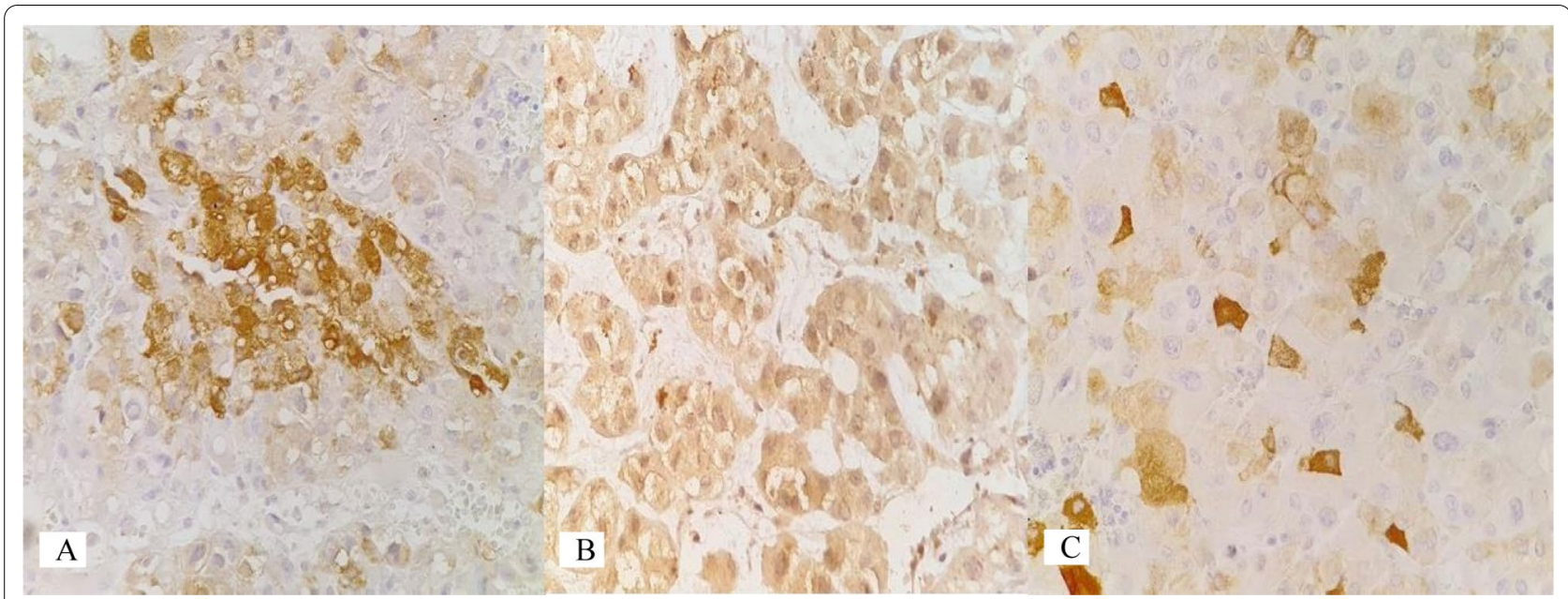

Fig. 2 Immunohistochemistry study. A Inhibin $\times 400$. B Melan A ×400. C Synaptophysin $\times 400$

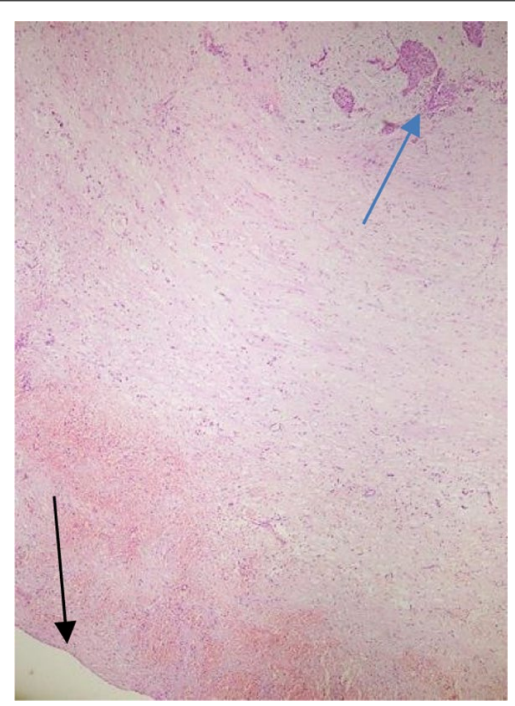

Fig. 3 Microscopy of the pancreas cyst showing no definite epithelial lining (black arrow) and remnant of the endocrine component (blue arrow)

symptoms, and during the 10-month follow-up visits, no clinical or radiologic deficits were noted. We requested and obtained the informed consent from the patient for publishing the case report and the publication of the accompanying images. Also, our institutional approval was not required to publish the case details.

\section{Discussion}

Our patient was a middle-age woman with multiple chronic diseases (ESRD and HTN), recurrent cyst of the pancreas, and a new periceliac mass, which was incidentally found by endoscopic ultrasound during post-partial pancreatectomy follow-up. Since the previous pathology report of the pancreas was consistent with mucinous neoplasm and regarding the malignant potential of mucinous cystic lesions, it was considered as a suspicious malignant lesion. Fortunately, on pathologic examination, the periceliac mass was an adrenal gland showing ACA associated with myelolipomatous metaplasia. During the 10-month follow-up visits, no related symptom or abnormal radiologic findings were noted.

Adrenal incidentalomas are clinically silent adrenal masses that are found during the investigation of unrelated problems. Myelolipoma is a lipomatous tumor, which is the second most common primary adrenal incidentaloma following ACAs, accounting for about 3\% of the incidentalomas [6,7]. Generally, adrenal lipomatous tumors are uncommon neoplasms. Myelolipoma is the predominant type, but other types of lipomatous adrenal gland tumors such as lipomas, teratoma, angiomyolipoma, and adrenocortical tumors with fat component (lipomatous and myelolipomatous metaplastic changes) are often described as case reports in the literature $[8,9]$. Among these lipomatous lesions, just myelolipoma and myelolipomatous metaplasia contain bone marrow elements, in addition to adipose tissue.

Adrenal myelolipoma was first described by Arnold as "adrenal lipoma" in 1866, but it was Gierke in 1905 who described the myeloid component. The term "myelolipoma" was coined by Oberling in 1929. Most of the early studies described it as an incidental autopsy finding or as occasional case reports. Despite its benign behavior, adrenal myelolipoma is clinically relevant as it might cause significant difficulties in differential diagnosis of other adrenal tumors $[9,10]$. Myelolipomatous metaplasia is a secondary degenerative change in a primary 
adrenal tumor. It usually occurs in ACA, which is the most common adrenal tumor. Due to increased availability, access to and use of abdominal imagery investigations [ultrasound scan, computed tomography (CT) scan and magnetic resonance imaging (MRI) scan] for the evaluation of various conditions and symptoms, cases of adrenal lipomatous tumors are now diagnosed more frequently [11].

In terms of pathogenesis, it seems that chronic adrenal stimulation, such as necrosis or inflammation due to cancer, Cushing's disease, HTN, diabetes, obesity, and stressful lifestyle could lead to metaplasia of the reticuloendothelial cells, which could lead to the development of adrenal myelolipomas. This hypothesis is supported by the increased incidence of the lesion in the advanced years of life. Another hypothesis claims that adipocytes develop from the mesenchymal stem cells in the endothelium; this results in inflammation causing the adrenal cortex to secrete mediators responsible for the recruitment of hematopoietic progenitors. The myelolipomatous metaplasia also occurs in response to stress related to stimuli such as necrosis, infection, stress, or trauma $[9,12-15]$. The metaplastic change in our case could be explained by chronic stress of ESRD, HTN, and the previous surgery.

Generally, there are no specific laboratory tests that would be diagnostic of adrenal myelolipoma, and in contrast to ACA, which could be functional or nonfunctional, most myelolipomas are silent. The National Institute of Health Consensus Panel on adrenal incidentaloma (2002) concluded that adrenal myelolipoma could be regarded as an exception to the mandatory metabolic work-up of a newly discovered adrenal mass [8-10]. It also makes sense that adrenal tumors with myelolipomatous metaplasia show metabolic changes that are compatible with the primary tumor [15-17].

Ultrasound is usually inadequate for the diagnosis of myelolipoma. On CT and MRI, the diagnosis of myelolipoma can be confidently made when an adrenal mass is composed of at least $50 \%$ fat. In larger masses with lesser amounts of fat, lipomatous or myelolipomatous degeneration of other adrenal tumors should be considered. On the other hand, the typical imaging features of adenoma in CT and MRI include small size, homogeneous and well-circumscribed, presence of intracellular lipid, and low-to-intermediate signal intensity on T2-weighted images. When ACAs show atypical features (that is, mixed areas of low and higher attenuation or heterogeneous signal intensity), the possibility of an adrenal collision tumor or secondary degenerative changes should be considered $[10,18]$.

On pathologic examination, it is easier to distinguish myelolipoma from an adrenocortical tumor with myelolipomatous metaplasia. In fact, myelolipoma is a well-demarcated combination of fat and myeloid tissue, while myelolipomatous metaplasia is usually present as scattered ill-defined foci of fat and myeloid tissue within another tumor.

In management of adrenal incidentalomas, European Society of Endocrinology Clinical Practice Guideline in collaboration with the European Network for the Study of Adrenal Tumors recommend determining if an adrenal mass is benign or malignant at the time of initial detection, which is just possible through CT scan and MRI study. Patients with an asymptomatic, nonfunctioning unilateral adrenal mass and obvious benign features on imaging studies do not need surgical intervention, whereas adrenalectomy has been suggested in patients with unilateral adrenal masses with radiological findings suspicious of malignancy (heterogeneous features and size $>4 \mathrm{~cm}$ ) [19].

Until now, a few adrenocortical tumors showing myelolipomatous metaplasia with full separated demographic and pathologic data have been reported (Table 1).

Although myelolipoma and myelolipomatous metaplastic change are very similar in pathogenesis, there are still some fine differences that help us make a more accurate diagnosis. Table 2 presents a comparison between myelolipoma and myelolipomatous metaplasia in terms of demography and pathology. The myelolipomatous metaplasia data have been extracted from our review.

Among 20 cases of myelolipomatous metaplasia that we reviewed, the primary adrenal pathology in 13 cases (65\%) was ACA (the most common adrenal tumor). Sixteen cases $(80 \%)$ were female, and just four cases $(20 \%)$ were male. This sex pattern could be justified by female predominance of ACA. The mean age was a little lower in myelolipomatous metaplasia, and the dominant side was left in contrast to myelolipoma (most cases are right sided). Common chronic diseases were HTN and DM in both groups. Myelolipoma is usually a well-defined mass with fat component that is easily seen grossly, while myelolipomatous metaplasia is an ill-defined microscopic finding. In addition to adrenocortical hyperplasia, adrenocortical carcinoma, adrenocortical neoplasm of uncertain malignant potential, and oncocytoma, some metaplastic changes were accompanied with vascular lesions such as endothelial cyst (two cases), cavernous hemangioma, and epithelioid angiosarcoma.

Adrenal tumors with myelolipomatous change may show heterogeneous appearance and could mimic malignancy even using advanced radiologic studies. Although in our case no further radiologic evaluation was done, regarding the recurrence of pancreatic cyst, the histology of the primary cyst, and also surgery protocols in approach to suspicious adrenal incidentalomas, finally 
Table 1 Demographic and pathologic data of adrenal tumors with myelolipomatous metaplasia

\begin{tabular}{|c|c|c|c|c|c|c|}
\hline No. & Authors & Age & Sex & Adrenal pathology & Underlying disease & Other remarkable finding \\
\hline 1 & Vyberg et al. [15] & 31 & $\mathrm{~F}$ & ACA & - & - \\
\hline 2 & Parenteau et al. [16] & 28 & M & Pigmented adrenal hyperplasia & - & - \\
\hline 3 & Lamas et al. [17] & 49 & $\mathrm{~F}$ & ACA & - & - \\
\hline 4 & Lamas et al. [17] & 67 & $\mathrm{~F}$ & ACA & HTN/DM & - \\
\hline 5 & Montone et al. [20] & 50 & $\mathrm{~F}$ & Adrenocortical neoplasm of UMP & - & - \\
\hline 6 & Montone et al. [20] & 56 & $\mathrm{~F}$ & Adrenocortical neoplasm of UMP & - & - \\
\hline 7 & Montone et al. [20] & 67 & $\mathrm{~F}$ & ACA & HTN & - \\
\hline 8 & Inomoto et al. [21] & 46 & $\mathrm{~F}$ & Black ACA & HTN & - \\
\hline 9 & Yamada et al. [22] & 72 & $\mathrm{~F}$ & ACA & Chronic hepatitis $C$ & Concurrent endothelial cyst in adrenal \\
\hline 10 & Shastri et al. [23] & 40 & $\mathrm{~F}$ & Oncocytoma & HTN & Bilateral \\
\hline 11 & Gurbuz et al. [24] & 66 & M & ACA & $\begin{array}{l}\text { Goiter surgery } \\
\text { Nephrectomy }\end{array}$ & - \\
\hline 12 & Yokoyama et al. [25] & 34 & $\mathrm{~F}$ & ACC & - & $\begin{array}{l}\text { Tumor site was ectopic adrenal (retroperi- } \\
\text { toneum) }\end{array}$ \\
\hline 13 & Chandramahanti et al. [26] & 66 & $\mathrm{~F}$ & ACA & HTN/DM & - \\
\hline 14 & Hendry et al. [27] & 60 & $\mathrm{~F}$ & ACA & HTN/hypothyroidism & $\begin{array}{l}\text { Concurrent epithelioid angiosarcoma in } \\
\text { adrenal }\end{array}$ \\
\hline 15 & Kinebuchi et al. [28] & 77 & M & Cavernous hemangioma & $\begin{array}{l}\text { History of gastric and } \\
\text { rectal cancer }\end{array}$ & - \\
\hline 16 & Dokmetas et al. [29] & 53 & $\mathrm{~F}$ & ACA & HTN/DM & Concurrent schwannoma in adrenal \\
\hline 17 & Larose et al. [30] & 61 & $\mathrm{~F}$ & Macronodular adrenal hyperplasia & - & Bilateral \\
\hline 18 & Firat et al. [31] & 62 & $\mathrm{~F}$ & ACA & - & - \\
\hline 19 & Firat et al. [31] & 73 & M & ACA & HTN & Concurrent endothelial cyst in the adrenal \\
\hline 20 & Current case & 45 & $\mathrm{~F}$ & ACA & $\begin{array}{l}\text { HTN } \\
\text { ESRD }\end{array}$ & Concurrent pancreas cyst \\
\hline
\end{tabular}

$A C A$ adrenocortical adenoma, $A C C$ adrenocortical carcinoma, UMP uncertain malignant potential, HTN hypertension, DM diabetes mellitus, ESRD end-stage renal disease

Table 2 Demographic and pathologic differences between myelolipoma [6] and myelolipomatous metaplasia

\begin{tabular}{lll}
\hline & Myelolipoma & Myelolipomatous metaplasia $^{\mathbf{a}}$ \\
\hline Dominant sex & No gender predilection & Female predominant \\
Age range, years (mean) & $41-84(62)[6]$ & $28-77(52.5)$ \\
Chronic disease & HTN/DM & HTN/DM \\
Dominant side & Right & Left \\
Gross and microscopic feature & Well-demarcated macroscopic fat & III-demarcated microscopic fat \\
Most common collision tumor & ACA & ACA \\
\hline
\end{tabular}

HTN hypertension, DM diabetes mellitus, ACA adrenocortical adenoma

a The mentioned data are according to current review

there was no change in the patient treatment, but it is very important to consider incidentalomas before operation to reduce surgical complications and it should be in the differential diagnosis when we deal with multiple tumors.

\section{Conclusion}

In a patient with a primary tumor, considering any new mass as malignancy should be avoided. Our case highlights the significance of complete evaluation of incidental findings by laboratory tests and radiologic studies before surgical intervention, even in the setting of another primary tumor. Based on observations in this case report and to the best of our knowledge, myelolipoma and myelolipomatous change (metaplasia) are two different entities. Although very similar considering pathogenesis, there are still some differences. In terms of epidemiologic and pathologic features, it appears that myelolipomatous metaplasia follows the primary adrenal tumor more than myelolipoma. 


\section{Acknowledgements}

The authors would like to thank Shiraz University of Medical Sciences, Shiraz, Iran and also Center for Development of Clinical Research of Nemazee Hospital and Dr. Nasrin Shokrpour for editorial assistance.

\section{Authors' contributions}

$\mathrm{AMH}, \mathrm{SN}$, and MM developed the concept of the study and the study design and wrote the draft manuscript. MM extracted the history. NS examined the patient and performed the surgery. AMH and SN with the input of all authors interpreted the patient data. AMH and SN reviewed and analyzed all data. All authors read and approved the final manuscript.

\section{Funding}

Not applicable.

\section{Availability of data and materials}

All data generated or analyzed during this study are included in this published article.

\section{Declarations}

\section{Ethics approval and consent to participate}

Our institutional approval was not required to publish the case details.

\section{Consent for publication}

A written informed consent was obtained by the patient for publishing the case report and the publication of the accompanying images. A copy of the written consent is available for review by the Editor-in-Chief of this journal.

\section{Competing interests}

The authors declare that they have no competing interests.

\section{Author details}

${ }^{1}$ Department of Pathology, Shiraz Medical School, Shiraz University of Medical Sciences, Shiraz, Iran. ${ }^{2}$ Department of Pathology, Shiraz Transplant Center, Abu Ali Sina Hospital, Shiraz University of Medical Sciences, Shiraz, Iran. ${ }^{3}$ Department of hepatopancreatobiliary and organ transplant surgery, Shiraz Transplant Center, Abu Ali Sina Hospital, Shiraz University of Medical Sciences, Shiraz, Iran. ${ }^{4}$ Department of Surgery, Shiraz Medical School, Shiraz University of Medical Sciences, Shiraz, Iran.

Received: 20 January 2021 Accepted: 31 May 2021 Published online: 04 July 2021

\section{References}

1. Mansmann G, Lau J, Balk E, et al. The clinically inapparent adrenal mass: update in diagnosis and management. Endocr Rev. 2004;25(2):309-40.

2. Mantero F, Terzolo M, Arnaldi G, et al. A survey on adrenal incidentaloma in Italy. JCE \& M. 2000;85(2):637-44.

3. Kerkhofs TM, Roumen RM, Demeyere TB, et al. Adrenal tumors with unexpected outcome: a review of the literature. Int J Endocrinol. 2015;710514:7.

4. Pinto A, Barletta JA. Adrenal tumors in adults. Surg Pathol Clin. 2015;8(4):725-49.

5. Hansel DE, Reuter VE. Adrenal pathology in the adult: a urological pathologist's perspective. Adv Anat Pathol. 2016;23(5):273-84.

6. Khater N, Khauli R. Myelolipomas and other fatty tumours of the adrenals. Arab J Urol. 2011;9(4):259-65.

7. Mantero F, Albiger N. A comprehensive approach to adrenal incidentalomas. Arq Bras Endocrinol Metabol. 2004;48(5):583-91.

8. Lam AK. Lipomatous tumours in adrenal gland: WHO updates and clinical implications. Endocr Relat Cancer. 2017;24(3):65-79.
9. Decmann Á, Perge P, Tóth M, et al. Adrenal myelolipoma: a comprehensive review. Endocrine. 2018;59(1):7-15.

10. Shenoy VG, Thota A, Shankar R, et al. Adrenal myelolipoma: controversies in its management. Indian J Urol. 2015;31(2):94-101.

11. Venyo AKG. Myelolipoma of the adrenal gland: a review and update of the literature. Pulsus J Surg Res. 2018;2(2):50-63.

12. Yildiz L, Akpolat I, Erzurumlu K, et al. Giant adrenal myelolipoma: case report and review of the literature. Pathol Int. 2000;50:502-4.

13. Han M, Burnett AL, Fishman EK, et al. The natural history and treatment of adrenal myelolipoma. J Urol. 1997;157:1213-6.

14. Bishop E, Eble JN, Cheng $L$, et al. Adrenal myelolipomas show nonrandom $\mathrm{X}$-chromosome inactivation in hematopoietic elements and fat: support for a clonal origin of myelolipomas. Am J Surg Pathol. 2006;30(7):838-43.

15. Vyberg M, Sestoft L. Combined adrenal myelolipoma and adenoma associated with Cushing's syndrome. Am J Clin Pathol. 1986;86(4):541-5.

16. Parenteau C, Mongeau CJ, Bénard B, et al. Pigmented adrenal hyperplasia with myelolipomatous changes and bilateral testicular enlargement in an untreated man with 21-hydroxylase deficiency. Endocr Pract. 2000;6(3):260-3.

17. Lamas C, López LM, Lozano E, et al. Myelolipomatous adrenal masses causing Cushing's syndrome. Exp Clin Endocrinol Diabetes. 2009;117:440-5.

18. Schieda N, Siegelman ES. Update on CT and MRI of adrenal nodules. AJR. 2017;208:1-12.

19. Fassnacht M, Arlt W, Bancos I, et al. Management of adrenal incidentalomas: European Society of Endocrinology Clinical Practice Guideline in collaboration with the European Network for the Study of Adrenal Tumors. Eur J Endocrinol. 2016;175:G1-34.

20. Montone KT, Rosen M, Siegelman ES, et al. Adrenocortical neoplasms with myelolipomatous and lipomatous metaplasia: report of 3 cases. Endocr Pract. 2009;15(2):128-33.

21. Inomoto $\mathrm{C}$, Sato H, Kanai G, et al. Black adrenal adenoma causing preclinical Cushing's syndrome. Tokai J Exp Clin Med. 2010;35(2):57-61.

22. Yamada S, Tanimoto A, Wang K, et al. Non-functional adrenocortical adenoma: a unique case of combination with myelolipoma and endothelial cysts. Pathol Res Pract. 2011;207:192-6.

23. Shastri C, Rana C, Kumari N, et al. Bilateral adrenocortical oncocytoma with bilateral myelolipomatous metaplasia. Endocr Pathol. 2012;23(2):112-4

24. Gurbuz E, Sayar H, Bakaris S, et al. Adrenal myelolipoma's connection with adenoma in the same adrenal gland. BMJ Case Rep. 2013. https://doi.org/ 10.1136/bcr-2013-008925.

25. Yokoyama H, Adachi T, Tsubouchi K, et al. Non-functioning adrenocortical carcinoma arising in an adrenal rest: immunohistochemical study of an adult patient. TJEM. 2013;229(4):267-70.

26. Chandramahanti S, Santhanam P, Taieb D. Myelolipomatous adrenal adenoma and adrenal scintigraphy: a case presentation and review of literature. J Endocrinol Metab. 2014;4(5-6):148-50.

27. Hendry $\mathrm{SH}$, Forrest $\mathrm{C}$. Epithelioid angiosarcoma arising in an adrenal cortical adenoma: a case report and review of the literature. Int J Surg Pathol. 2014;22(8):744-8.

28. Kinebuchi Y, Daimon H, Kawaguchi K. Adrenal cavernous hemangioma associated with myelolipomatous metaplasia. Int J Urol. 2016;23(1):106-8

29. Dokmetas HS, Hıncal HO, Ozdenkaya Y, et al. A rare incidentaloma: adrenal schwannoma. Endocr Abstr. 2018;56:39.

30. Larose S, Bondaz L, Mermejo LM, et al. Coexistence of myelolipoma and primary bilateral macronodular adrenal hyperpla sia with GIP-dependent Cushing's syndrome. Front Endocrinol. 2019;10:618.

31. Fırat C, Eryiğit S, Yener $\mathrm{S}$, et al. Myelolipomatous changes within adrenocortical adenoma. Cukurova Med J. 2019:44(3):1135-8.

\section{Publisher's Note}

Springer Nature remains neutral with regard to jurisdictional claims in published maps and institutional affiliations. 